\title{
On Design of Intelligent Load-Frequency Controllers Considering Variation of Parameters and Generation Rate Constraint
}

\author{
Khoat Nguyen \\ Faculty of Control and Automation, Electric Power University, 235 Hoang Quoc Viet Str., Hanoi, Vietnam, khoatnn @epu.edu.vn
}

\begin{abstract}
The application of artificial intelligence (AI) together with biology-inspired optimization techniques in designing control strategies has recently gained significant achievements for engineers. The integration of AI and meta-heuristic optimization mechanisms forms effective hybrid controllers. This investigation focuses on the design of such hybrid controllers especially applied for load-frequency control (LFC) which was considered to be one of the most important control strategies in a hydroelectric power plant. The proposed hybrid LFC controller is formed by two phases: an effective evolutionary optimization algorithm named artificial bee colony (ABC) and a fuzzy logic architecture. The applicability and robustness of the proposed LFC control methodology will be compared to conventional regulators under unfavorably practical conditions of the hydropower system, i.e. the occurrence of parameter variations and the generation rate constraint. These conditions usually affect control performances of a system, and thus their impacts need to be strictly minimized. Simulation results implemented in MATLAB software will demonstrate the success of applying the hybrid LFC controllers proposed in this study.
\end{abstract}

Key words: LFC, hydropower system, $\mathrm{ABC}$ optimization technique, conventional and intelligent LFC controllers.

\section{INTRODUCTION}

In a power system, loads depending only upon users always vary over time. Such a random and continuous variation of loads usually causes an imbalance between generation and demand in power systems. Resulting from this phenomenon, the system frequency may be deviated from the nominal value $(50 \mathrm{~Hz}$ or $60 \mathrm{~Hz})$, affecting a lot of electric equipment. Therefore, designing a control strategy to eliminate this variation of loads should be absolutely considered to be a major control problem of power system's stability and operation. Technically, such a control problem has been named load-frequency control (LFC) [1-6].
It is the fact that hydropower systems are currently popular in a huge number of countries around the world, therefore, the LFC problem applied for these systems should be strictly taken into account. One of the most important goals of the LFC strategy is to maintain the system frequency at nominal value, thereby bringing the power utility back to stable state after the appearance of the random load variation. Such a goal directly relates to the speed control of synchronous generators in the hydropower plant. It is also considered to be a control problem of hydraulic turbines in the power system because of the close relationship between these turbines or prime movers and the electrical synchronous generators [4]. There is no doubt that the LFC problem of a hydroelectric power plant has been facing a lot of difficulties. Two of them include a variation of parameters and an appearance of generation rate constraint (GRC) beside the random load changes as mentioned earlier. The hydraulic turbine having an important factor, namely water starting time, is able to significantly affect dynamic characteristics of such a prime mover. Moreover, the GRC corresponding to specific types of turbines also impacts on the operation of the hydraulic turbine [6-7]. Besides, the variation of generator's parameters may usually occur during power system's operation. This is caused by unavoidable factors in such a hydroelectric power system such as temperature variability and frictions. As a result, these factors should be significantly considered when dealing with the LFC problem of a hydroelectric power plant.

At the beginning, this study delivers a concentration on the modelling of a hydropower plant following the LFC problem. It is noted that the variation of parameters of hydraulic turbines and synchronous generators together with the GRC will also be taken into account for this mathematical model. Then, the paper presents an overview of the artificial bee colony optimization method considered to be one of the most effective bio-inspired optimization techniques. Such a well-known optimization mechanism will be applied to design the LFC strategy proposed in this work. Specifically, it is employed to successfully determine significant factors of conventional LFC regulators (Integral and PID) as well as scaling factors of a fuzzy logic - based intelligent LFC controller. A meaningful simulation comparison between these LFC controllers will be implemented, verifying the effectiveness of the proposed control strategy. Conclusions regarding this study will also be provided to claim that the 
impact of parameter variation as well as the GRC in a hydroelectric power utility has been successfully minimized when applying the proposed control methodology.

\section{MODELLING OF AN ISOLATED HYDROPOWER PLANT FOR LOAD-FREQUENCY CONTROL 2.1 Typical model of an isolated hydropower system}

It is clear that a hydropower plant is supplied by a prime source of kinetic energy of water. A typical hydropower system model is depicted in Figure 1. Technically, such a model consists of several main units: a speed governor, valves/gates, a hydraulic turbine, and a synchronous generator [1-3]. To build a mathematical model of the isolated hydropower plant shown in Figure 1, it is necessary to establish the transfer functions for all components. The following will present a step-by-step method to mathematically model such a hydroelectric power plant.

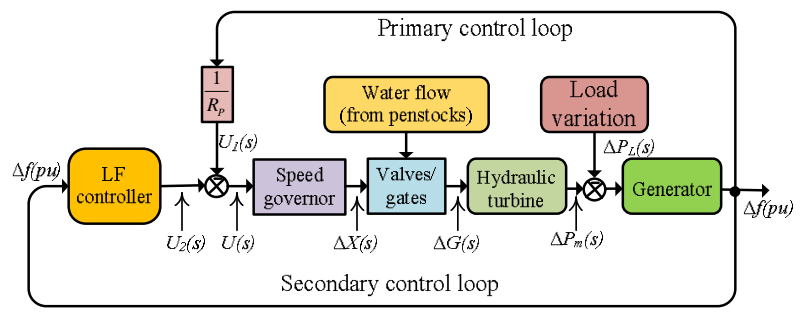

Figure 1: A typical model of isolated hydropower plants

\subsection{Establishment of the hydraulic turbine transfer function considering parameter variation}

To mathematically model a hydraulic turbine, let us consider a typical schematic of a hydropower utility. Normally, it contains a forebay or a reservoir, a penstock, a wicket gate, a water turbine and a synchronous generator. In [1], with a number of assumptions, the transfer function of a hydraulic turbine using linearizing method around an equilibrium point e.g. steady-state operating point is expressed below:

$$
\mathrm{W}_{t}(s)=\frac{\Delta P_{m}(s)}{\Delta g(s)}=\frac{1-T_{\mathrm{w}} s}{1+0.5 T_{\mathrm{w}} s}
$$

Where $T_{W}$ denotes the water starting time which can be calculated as:

$$
T_{W}=\frac{L U_{0}}{a_{g} H_{0}}
$$

Where $U_{0}$ and $H_{0}$ denote steady-state operating values of water velocity in the penstock and hydraulic head at gate, respectively. Meanwhile, $L$ and $a_{g}$ are the length of conduit and acceleration due to gravity, respectively. It should be noted that the time factor $T_{w}$ can be understood as the time for the water at standstill state to accelerate to a state with velocity $U_{0}$ under the effect of a water column with the head $H_{0}$.
One of the most difficult problems when designing a load-frequency control strategy is that the time coefficient $T w$ varies with load. Such a $T w$ normally belongs to an interval of $0.5 \mathrm{~s}$ and $4.0 \mathrm{~s}$ at a state of full load [1]. Figure 2 shows the dynamic responses of a hydraulic turbine with different water starting times. Absolutely, these responses regarding the step and impulse ones are different for various water starting times, making the design of speed controller highly challenging.

Beside the variation of the water starting time, in a hydropower utility, it is a fact that generation rate constraint (GRC) is able to affect the speed control scheme. According to [7], the GRC is a nonlinear function, which mainly aims to restrict the generation of power systems including hydroelectric power plants. An important characteristic of this function is that it is completely different for each category of power systems. For instance, with the hydropower plant, existed reports have provided a range of generation rates from $-360 \%$ to $270 \%$ in accordance with the lowering and rising generation phenomena [7]. Figure 3 depicts the effect of such a GRC function to the dynamic characteristics of a hydraulic turbine. Due to the nonlinearity of such a function, it is clear the impact on the LFC strategy should not be omitted and must be eliminated is this perspective. Section 3 will provide a control strategy applying an effective optimization mechanism to tackle this issue.
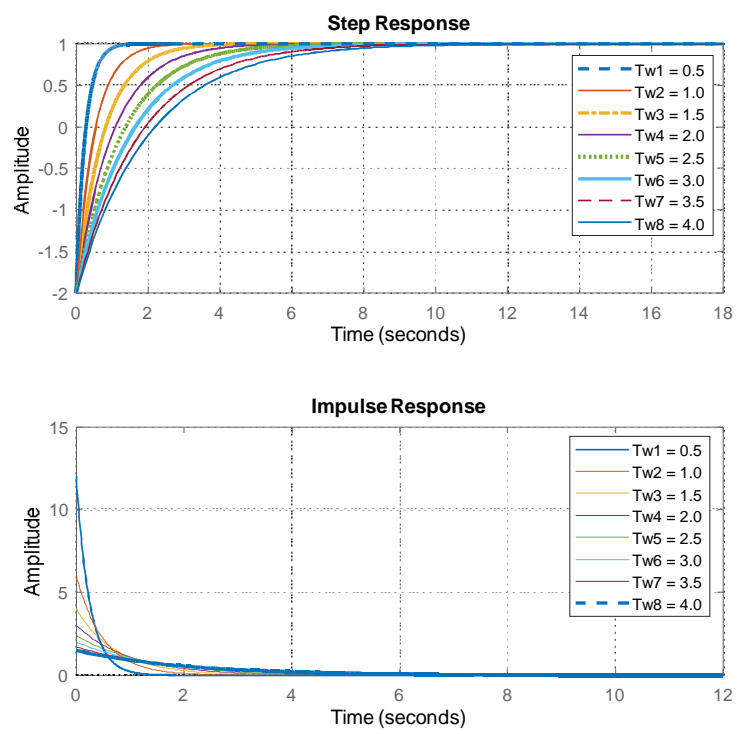

Figure 2: Dynamic characteristics regarding the step and impulse responses of the hydraulic turbines considering parameter variation 

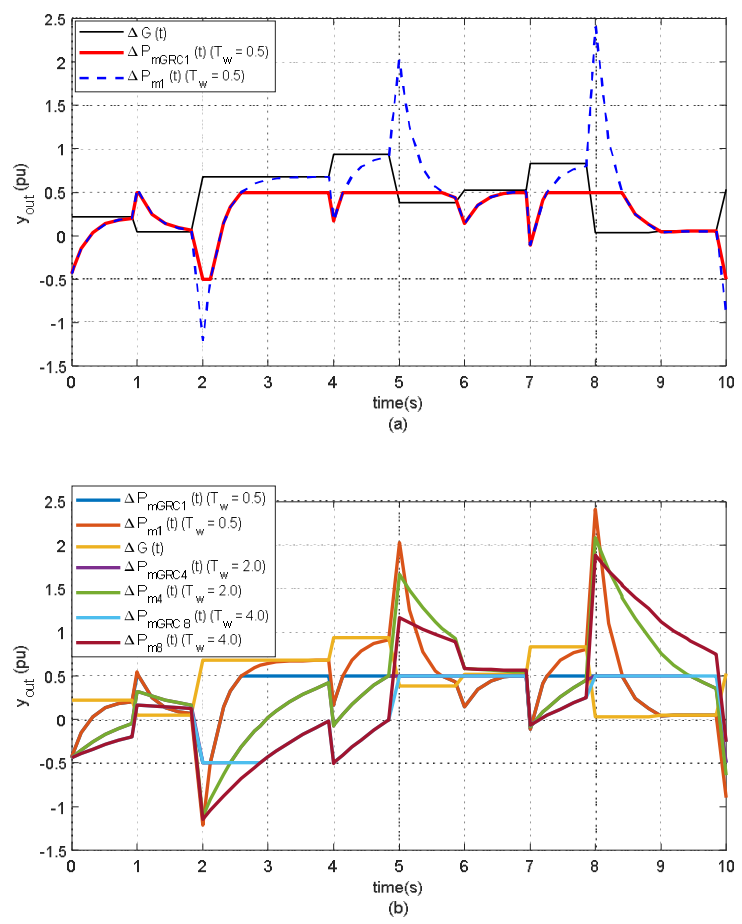

Figure 3: Effect of the water starting time variation and the generation rate constraint - GRC

\subsection{Mathematical model of a synchronous generator considering parameter variation}

A mathematical model of synchronous generators applied to the LFC problem has been studied in existed reports [1-3]. It is found that in response to the load change due to users the transfer function of a synchronous generator can be expressed as:

$$
\mathrm{W}_{\text {generator }}(s)=\frac{1}{2 H . s+D}=\frac{1}{M . s+D}=\frac{K_{P}}{T_{P} . s+1}
$$

Where $H$ denotes the synchronous generator inertia, meanwhile, $D$ is the load-damping coefficient depending upon the loads changing with frequency variation in the power system. These two factors, $M$ and $D$ in an ideal scenario, can be considered to be constant values. However, in practice, they can be deviated from the nominal values due to several reasons, e.g. operating temperature and friction. In this perspective, the variation of such two factors can affect the dynamic feature of the synchronous generator as shown in Figure 4. This may also impact on the efficiency of the LFC strategy embedded for a power system and thus such a variation must be minimized.
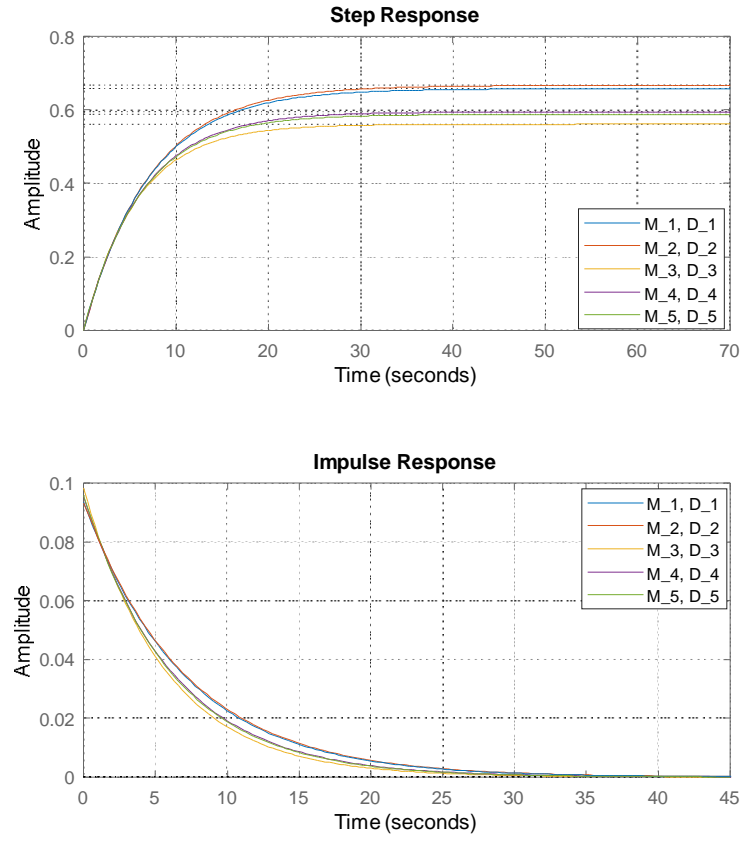

Figure 4: Step and impulse responses of synchronous generators considering parameter change

\subsection{Transfer functions of the speed governors and valves/gates}

A speed governor in a hydropower plant as its name has a fundamental functionality of regulating hydraulic turbine's speed due to load variation. Technically, each generator in a power system is usually equipped with a speed governor for such a speed control goal. To execute this mission, the primary control loop has been established as illustrated in Figure 1. It should be noted from this Figure that the feedback factor $R p$ characterizing the steady-state droop factor of the speed governor, which can be computed depending upon each governor. In this paper, it has been selected to be $5 \%$ as introduced in [1]. The transfer function used for such a speed governor is considered to be a first - order function below:

$$
\mathrm{W}_{g}(s)=\frac{\Delta X(s)}{U(s)}=\frac{1}{1+T_{g} s}
$$

The valves or gate servo forming the transient droop compensator is mathematically modelled as [1]:

$$
\mathrm{W}_{\text {gate }}(s)=\frac{\Delta G(s)}{\Delta X(s)}=\frac{1+T_{R} s}{1+T_{H} s}
$$

Where the factor $T_{H}$ can be calculated as expressed below:

$$
T_{H}=\frac{R_{T}}{R_{P} T_{R}}
$$

It should also be noted that both parameters $R_{T}$ and $T_{R}$ are varied depending upon the water starting time factor $T_{W}$ and inertia value $H$ of the generator as indicated below [1]: 


$$
\left\{\begin{array}{l}
R_{T}=\left[2.3-\left(T_{W}-1.0\right) * 0.15\right] \frac{T_{W}}{2 H} \\
T_{R}=\left[5.0-\left(T_{W}-1.0\right) * 0.5\right] T_{W}
\end{array}\right.
$$

In conclusion, most parameters as presented in equations (1)-(7) are changed over time in practice, making the design of LFC strategy highly challenging. As a result, finding an effective solution for this problem plays an important role from this point of view.

\subsection{Artificial bee colony optimization technique}

Considered to be one of the most effective bio-inspired optimization techniques, the artificial bee colony (ABC) method depending upon intelligent-biological behavior of honey bees is able to be applied for a huge class of control problems. In [8-12], the ABC algorithm describing a colony of artificial bees consisting of three groups, namely employed bees, onlookers and scouts. Employed bees are assigned to find the food source, then they go back to see onlookers waiting to make decision to choose the food source through dancing activities of the employed ones. Meanwhile, a scout is a bee whose food source has been abandoned by the onlookers. The flowchart describing the ABC method is depicted in Figure 5. There are several main steps for such an $A B C$ technique as presented below [9]:

- Initialization. Assuming that the number of employed bees $N_{e}$ are equal to the food sources. It is necessary to define the colony size or population $n_{P o p}$ and maximum number of iterations $N_{\max }$ which can be considered to be one of the termination criteria in this step. Besides, the number of variations together with the constraint are initialized.

- Implementation of iterations. There are three phases in accordance with three foresaid categories of the bee colony. Remember that a fitness or cost function needs to be defined first to check for every phase of the iterations. Here, when applied for the LFC of the hydroelectric power plant, the cost function has been selected as:

$$
J=\int_{0}^{T}|\Delta f(t)| t d t=I T A E \rightarrow \min
$$

Where $T$ is the simulation time. The cost function presented in (7) refers to ITAE (integral of time-weighted absolute error) which is a typical criterion to evaluate control performances of a control system. Using this control criterion, the system frequency deviation may force to zero in an acceptable steady-state time.

To execute the repeat of the $\mathrm{ABC}$ algorism, an updating expression is used as follows:

$$
V_{i j}=X_{i j}+\varphi_{i j}\left(X_{i j}-X_{k j}\right)
$$

Where $X$ denotes a vector of food sources or solutions of the optimization problem. Every time the vector $V$ is created as a candidate of new source position which will be evaluated by the fitness or cost function as presented in (8). The factor $\varphi_{i j}$ is a random number belonging to the interval $[-1 ; 1]$, showing the random selection of food sources for the honey bees.

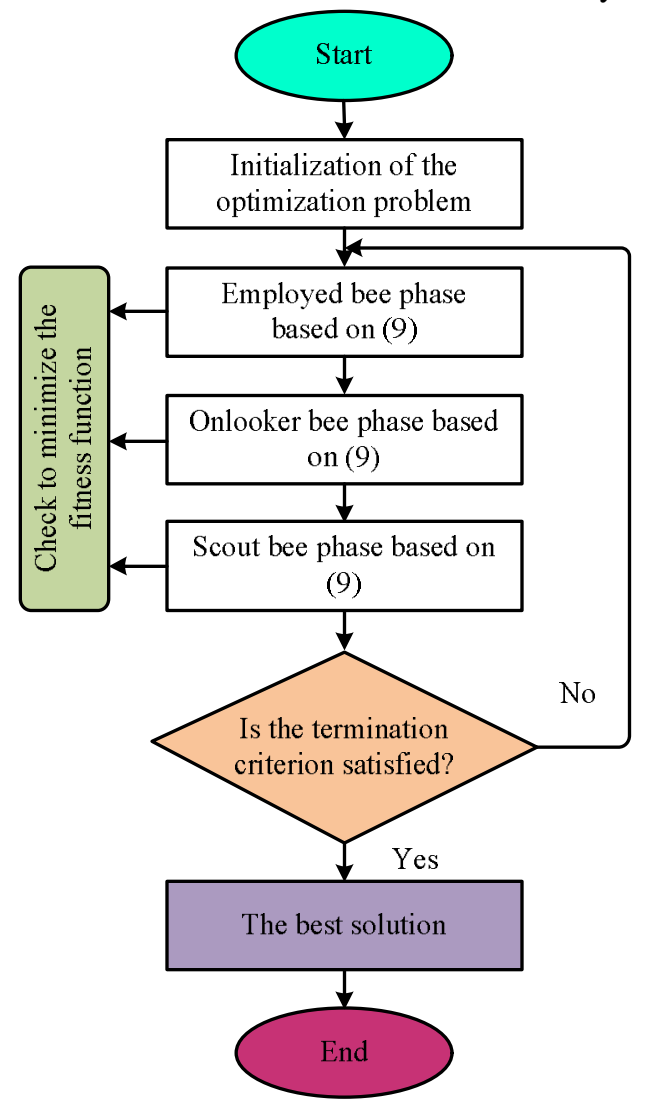

Figure 5: The flowchart of the $\mathrm{ABC}$ optimization technique

- Check the termination criterion. A typical termination criterion regarding the maximum number of the iterations of the $\mathrm{ABC}$ algorithm or an acceptable fitness function. If such a criterion is met, the best solution for the optimization problem is obtained.

The next section presents the application of the $\mathrm{ABC}$ algorithm for the LFC strategy of a hydropower plant.

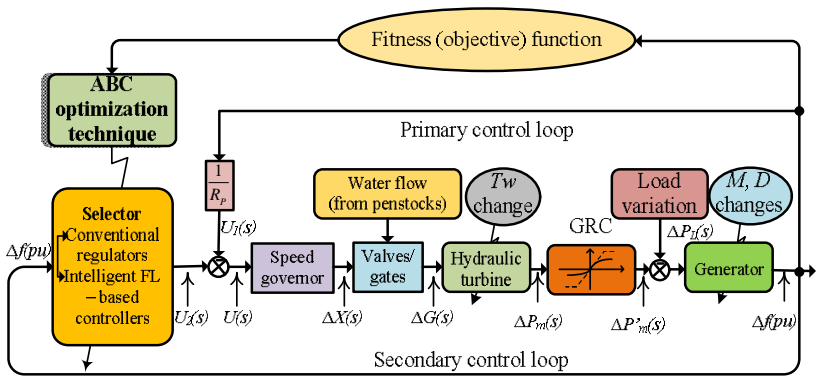

Figure 6: Proposed speed control strategy for a two-area interconnected hydropower system

\section{LOAD - FREQUENCY CONTROLLERS APPLYING ABC ALGORITHM}

The evolutionary - based optimization method like the ABC technique as mentioned earlier can be applied to design an effective control strategy. The LFC problem of a hydroelectric power plant is able to be considered to be a typical candidate. 
Figure 6 illustrates the control strategy based on the ABC algorithm. As shown, the hydropower plant considering nonlinearity of GRC function as well as the variation of parameters as mentioned in the previous section. The selector has the mission of choosing the LFC controllers. The conventional regulators including Integral and PID ones are able to eliminate the steady-state error, thus they have been used at first for solving the LFC problem [13-15]. These regulators have factors, such as integral, proportional and derivative which need to be determined. The $\mathrm{ABC}$ algorithm as presented in the previous section is completely able to optimize these coefficients, thereby improving control quality of the system.

The intelligent controllers such as fuzzy logic technique based ones have also been considered to be successful LFC candidates. This study introduces a 49-rule $2 / 1-\mathrm{I} / \mathrm{O}$ fuzzy logic architecture to deal with the LFC problem. This model consists of two inputs and one output with standard membership functions as shown in Figures 7-8. Besides, it uses 49 fuzzy logic rules in "IF...THEN..." form with an extracted representation of 20 rules as shown below [5].

'1. If ( $e$ is NB) and (delta_e is NB) then ( $u$ is NB) (1)'

'2. If (e is NB) and (delta_e is NM) then ( $u$ is NB) (1) "

'3. If (e is NB) and (delta_e is NS) then (u is NB) (1) '

'4. If (e is NB) and (delta_e is ZE) then ( $u$ is NB) (1) '

'5. If (e is NB) and (delta_e is PS) then ( $u$ is NM) (1)'

'6. If (e is NB) and (delta_e is PM) then ( $u$ is NS) (1) "

'7. If (e is NB) and (delta_e is $P B$ ) then ( $u$ is ZE) (1)'

'8. If (e is NM) and (delta_e is NB) then ( $u$ is NB) (1) '

'9. If (e is NM) and (delta_e is NM) then ( $u$ is NM) (1)'

'10. If (e is NM) and (delta_e is NS) then ( $u$ is NM) (1)'

$$
\text { ..... }
$$

'40. If (e is PM) and (delta_e is PS) then (u is PM) (1)'

'41. If (e is PM) and (delta_e is PM) then (u is PM) (1)'

'42. If (e is $P M)$ and (delta_e is $P B$ ) then ( $u$ is $P B$ ) (1)'

'43. If (e is $P B$ ) and (delta_e is NB) then ( $u$ is ZE) (1)'

'44. If (e is $P B$ ) and (delta_e is NM) then ( $u$ is $P S)(1)$ '

'45. If (e is $P B)$ and (delta_e is NS) then (u is PM) (1)'

'46. If (e is $P B$ ) and (delta_e is ZE) then ( $u$ is $P B$ ) (1)'

'47. If (e is $P B)$ and (delta_e is $P S$ ) then ( $u$ is $P B$ ) (1)'

'48. If (e is $P B$ ) and (delta_e is $P M$ ) then (u is $P B)(1)$ '

'49. If (e is $P B$ ) and (delta_e is $P B$ ) then (u is $P B$ ) (1)'

The illustration of $3 \mathrm{D}$ surface corresponding to the above rules is plotted in Figure 9.

It is also noted that in the studied fuzzy logic model, there are three scaling factors, two for the inputs and one for the output, need to be determined in order to optimize the control performance of the system. This issue is definitely able to be efficiently solved by applying the ABC mechanism. The next section will present simulation results to verify this confirmation.
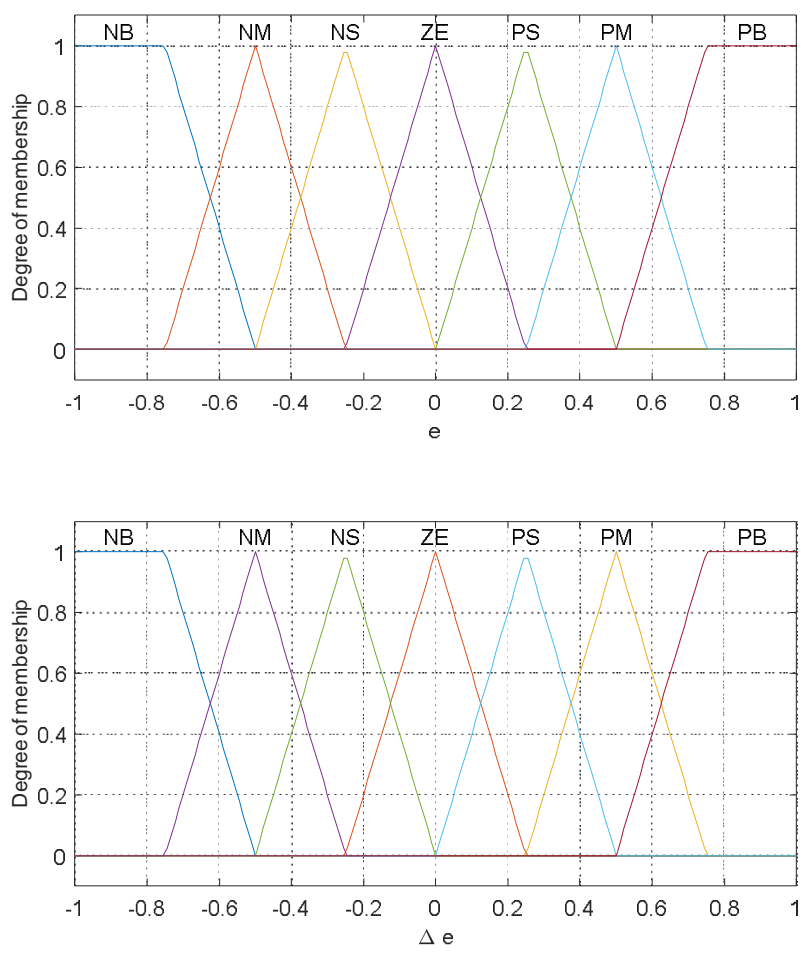

Figure 7: Standard degree membership functions for the two inputs of the fuzzy logic model

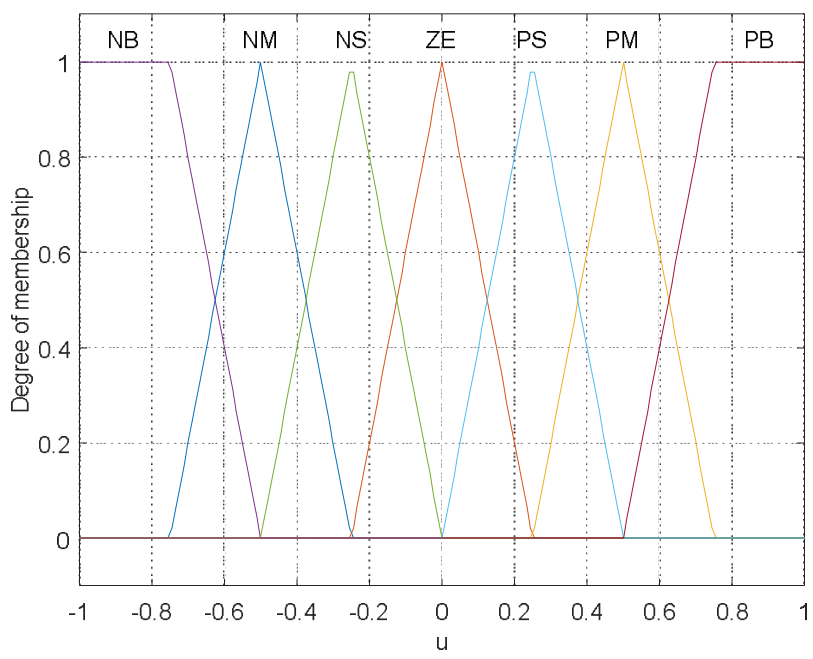

Figure 8: Standard degree membership functions for the output of the proposed fuzzy logic architecture 


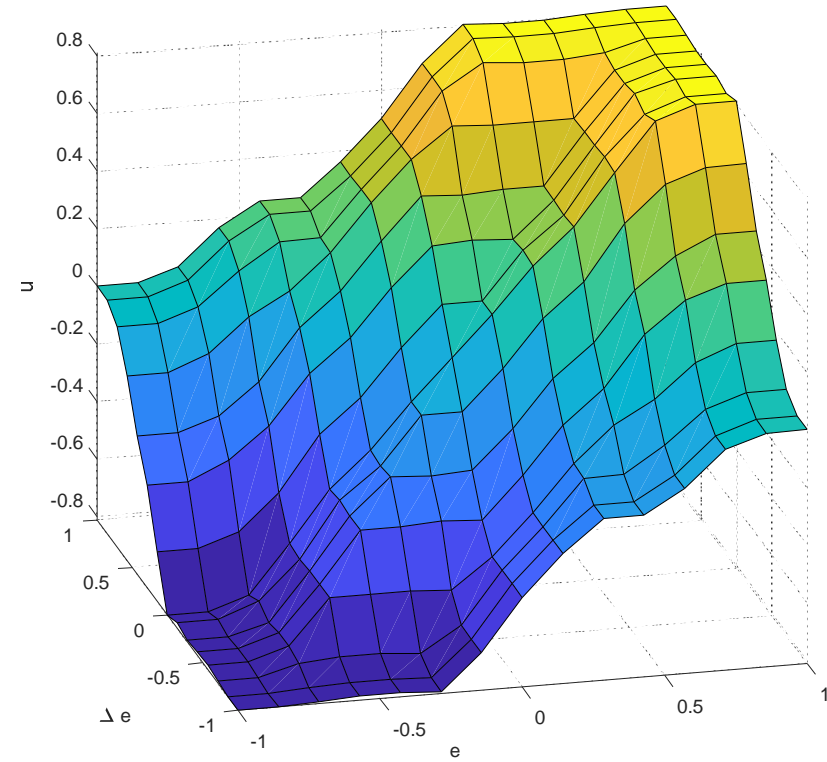

Figure 9: A 3-D surface illustration of the proposed fuzzy logic architecture

\section{SIMULATION RESULTS AND DISCUSSIONS}

Based on the LFC strategy proposed in Figure 6, a simulation model has been built in MATLAB/Simulink package version 2017b. Three foresaid LFC controllers are taken into account: two conventional regulators (Integral and PID) and one fuzzy logic counterpart. A random and continuous load change over time is embedded to the power system model as shown in Figure 10. This example of load variation is highly suitable for the practical load change which depending only upon users. Simulation parameters are presented in Appendix of this study.

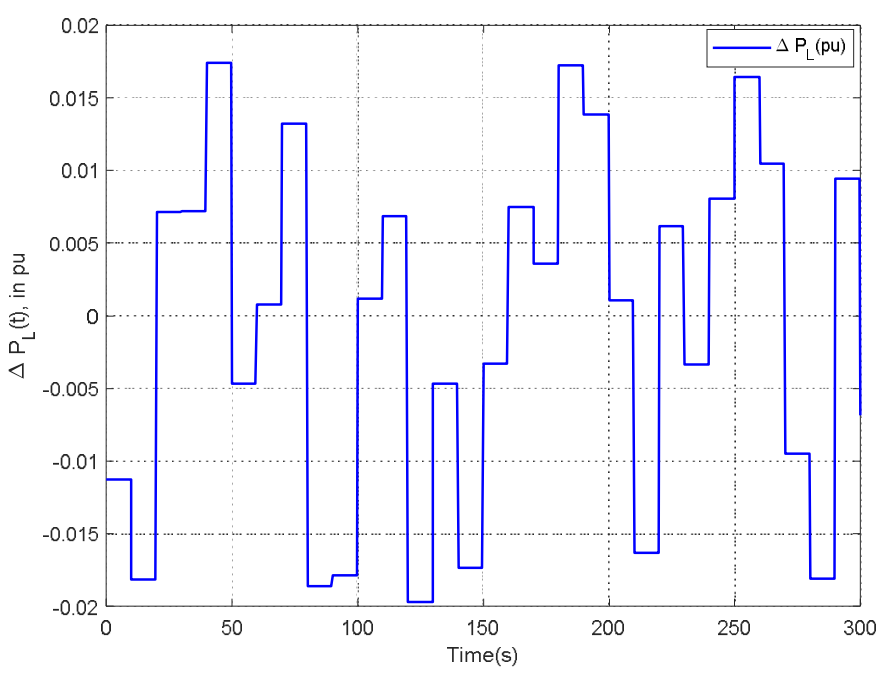

Figure 10: An illustration of random load change applied for the simulation process

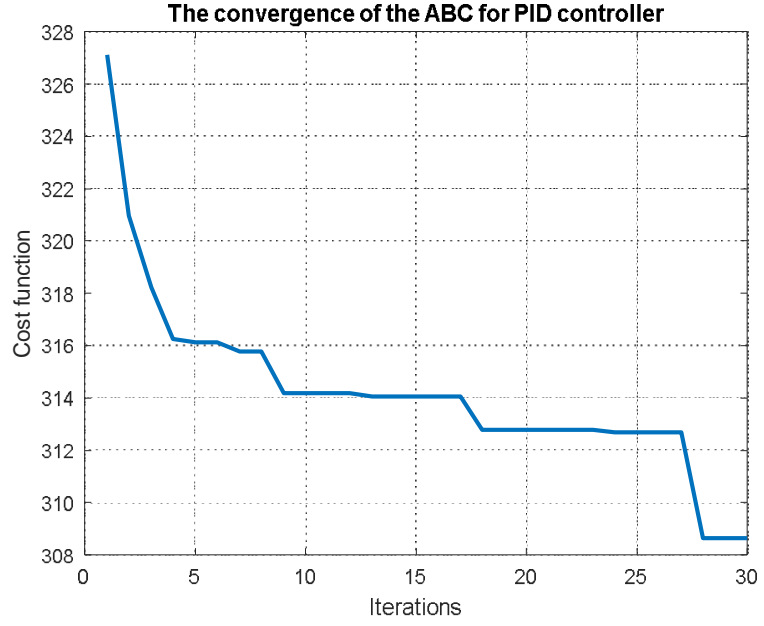

Figure 11: The convergence of the $\mathrm{ABC}$ for the $\mathrm{PID}$ controller

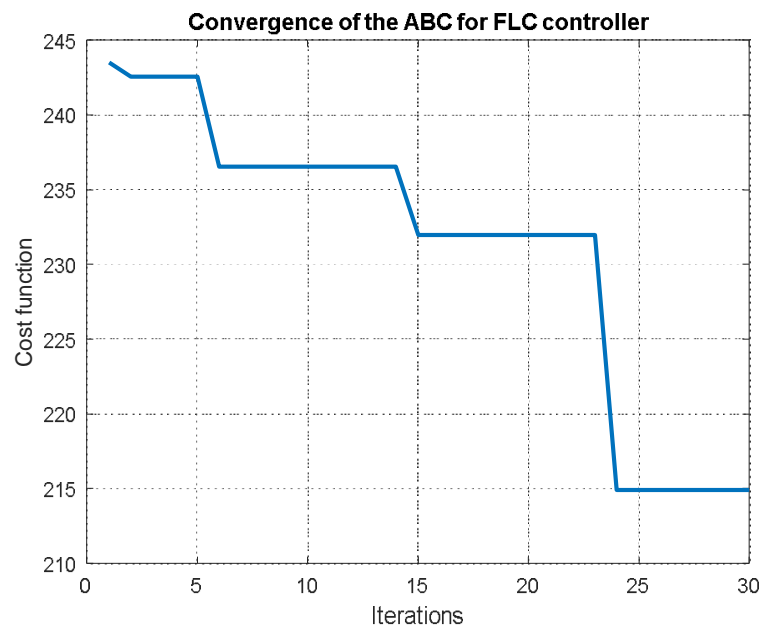

Figure 12: The convergence of the $\mathrm{ABC}$ for the fuzzy logic - based LFC controller

The simulation has been initialized by executing the $\mathrm{ABC}$ algorithm to optimally determine several parameters as foresaid. For instance, three factors including $K p, K i$ and $K d$ of the PID have been optimized with the cost function shown in Figure 11. Meanwhile, three scaling factors of the fuzzy logic-based controller are also determined, resulting the cost function as presented in Figure 12.

To evaluate the applicability of the proposed LFC controllers as well as compare their effectiveness, the dynamic responses of state variables should be plotted as shown in Figures 13-17. Figure 13 shows the dynamics of the hydropower plant using the ABC-based integral LFC regulator. Figure 14 and Figure 15 depicture the responses for the PID regulator and fuzzy logic counterpart, respectively. Overall, it can be found that the last two ones are much better than the first regulator. Since the most important role of the LFC is to maintain the system frequency at the nominal value to re-stabilize the control plant after the random load change occurrence, let us 
deeply consider the dynamic responses of frequency deviations. Figure 16 illustrates a comparison of the three LFC controllers. Obviously, the control quality of the PID as well as the intelligent controllers are much better than that of the integral counterpart. To evaluate the difference between the PID and the fuzzy logic controllers, Figure 17 shows the cost functions for these three LFC controllers. As shown, the fuzzy logic one is somewhat better than the PID regulator, thus both of them can be selected as feasible candidates for solving the LFC problem of a hydroelectric power plant.

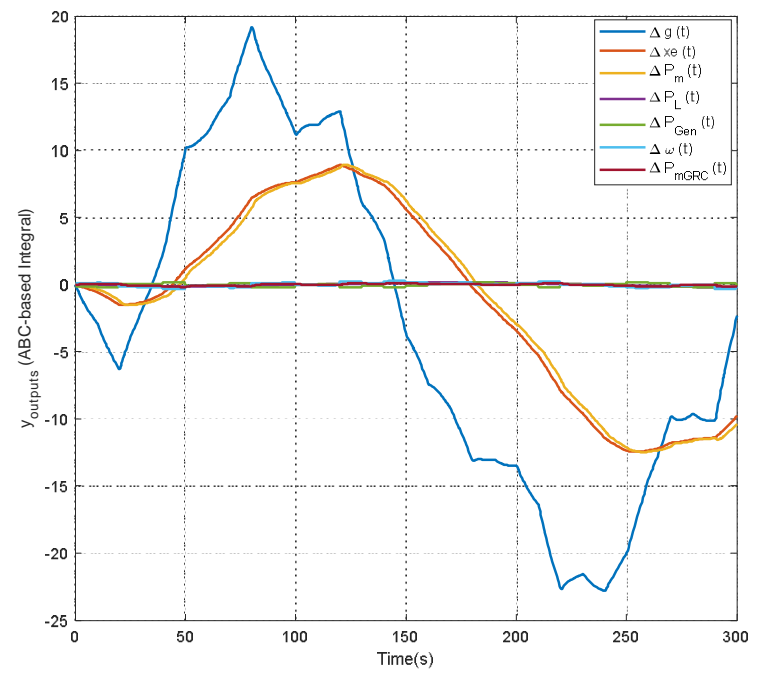

Figure 13: Dynamic response of the ABC-based integral controller

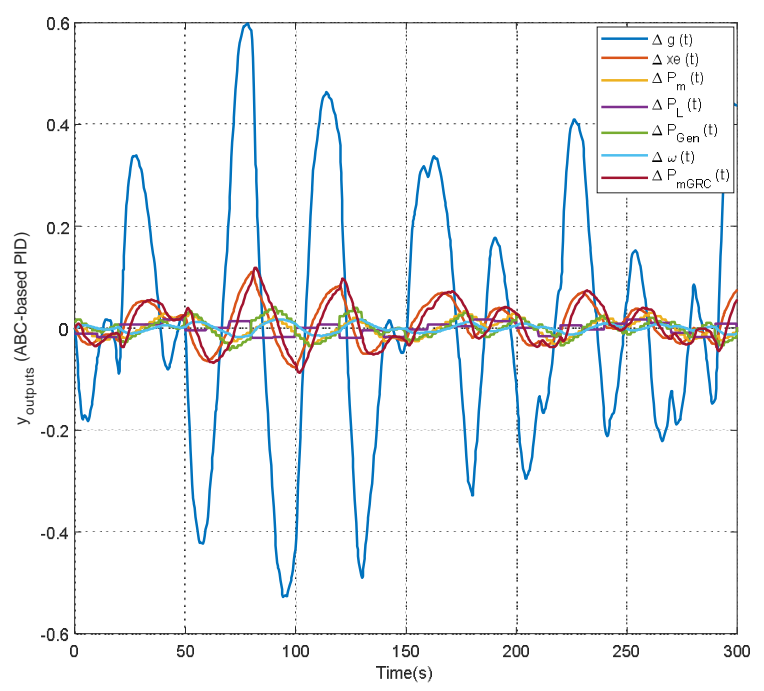

Figure 14: Dynamic response of the ABC-based PID controller

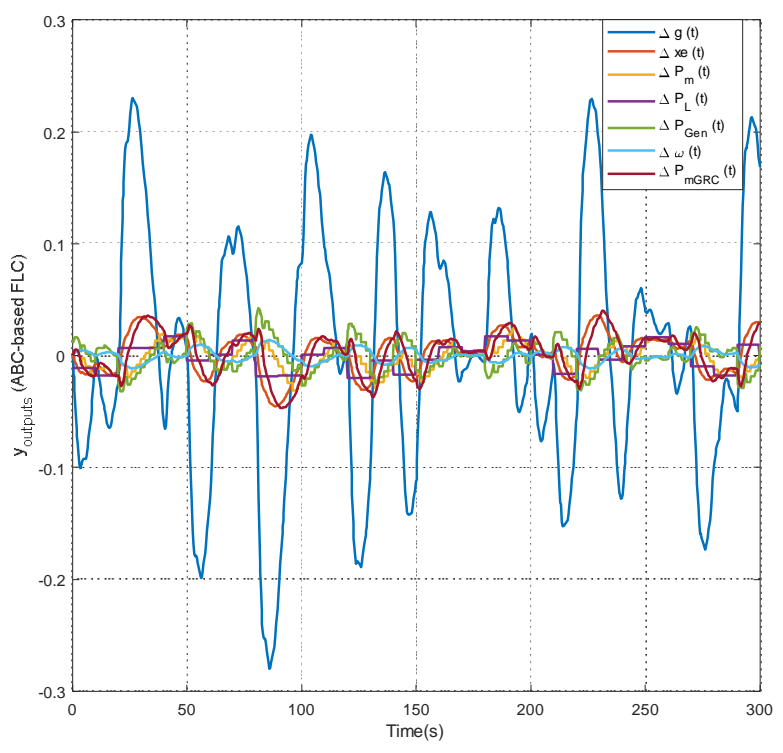

(a)

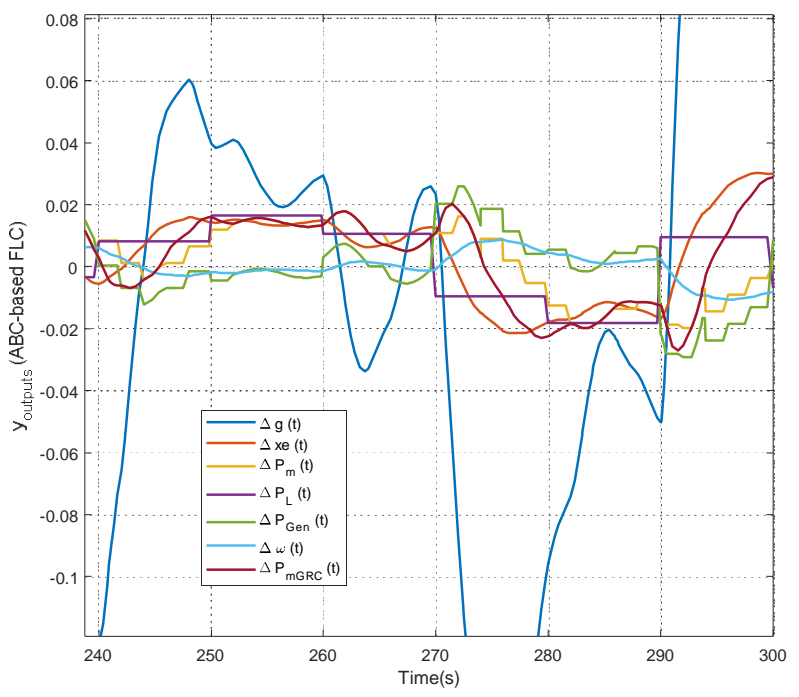

(b)

Figure 15: Dynamic response of the $\mathrm{ABC}$-based integral controller (a) An entire illustration

(b) An enlarged portion of the entire illustration 

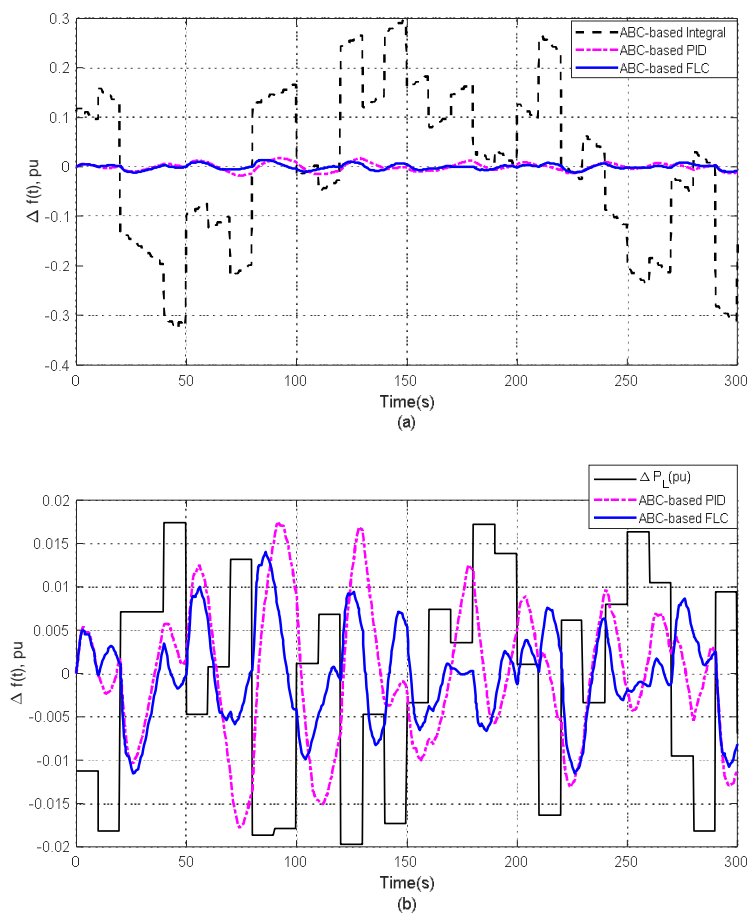

Figure 16: Comparison of the three LFC controllers based on the $\mathrm{ABC}$ algorithm regarding the system frequency deviations

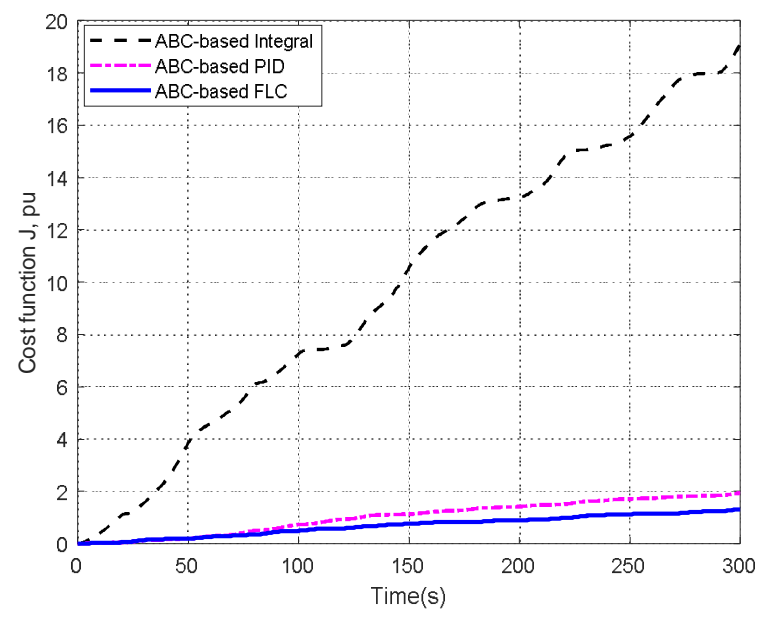

Figure 17: Cost or fitness functions of the three LFC controllers

\section{CONCLUSIONS AND FUTURE WORK}

An investigation regarding the design of different LFC controllers in a hydroelectric power plant applying the $\mathrm{ABC}$ optimization technique has been implemented in this study. These LFC controllers including Integral, PID and fuzzy logic are designed to deal with the nonlinearity of the GRC factor as well as the variation of parameters of elements in the hydropower utility. It was found that the flexible optimization of the $\mathrm{ABC}$ mechanism has been able to successfully tackle the foresaid problems. Simulation results obtained by
MATLAB/Simulink package also demonstrated the superiority of the ABC-based fuzzy logic LFC controller over the other two remaining LFC regulators.

Future work arising from this study should be further improvement of the control performances regarding the proposed ABC-based fuzzy logic FLC controllers. In this perspective, the membership functions together with the fuzzy logic rules will be optimized applying the $\mathrm{ABC}$ technique-one of the most effective bio-inspired optimization methods.

\section{ACKNOWLEDGEMENT}

The author wishes to thank Dr. Mai Phuong from Hanoi University of Industry for her meaningful discussion of the $\mathrm{ABC}$ algorithm.

\section{APPENDICES}

\section{A. Nomenclature}

\begin{tabular}{|l|l|l|l|}
\hline No & Symbol & Description & Unit \\
\hline $\mathbf{1}$ & $T_{g}$ & Time constant of speed governor & second \\
\hline $\mathbf{2}$ & $T_{w}$ & Water starting time & second \\
\hline $\mathbf{3}$ & $L$ & Length of conduit & $\mathrm{m}$ \\
\hline $\mathbf{4}$ & $A$ & Pipe area & $\mathrm{m}^{2}$ \\
\hline $\mathbf{5}$ & $a_{g}$ & Acceleration due to gravity & $\mathrm{m} / \mathrm{s}^{2}$ \\
\hline $\mathbf{6}$ & $f$ & Frequency & $\mathrm{pu}$ \\
\hline $\mathbf{7}$ & $\omega$ & Rotational speed of generator & $\mathrm{pu}$ \\
\hline $\mathbf{8}$ & $H$ & Inertia of generator & $\mathrm{kg} \cdot \mathrm{m}^{2}$ \\
\hline $\mathbf{9}$ & $M$ & Twice of generator inertia & $\mathrm{kg} \cdot \mathrm{m}^{2}$ \\
\hline $\mathbf{1 0}$ & $D$ & Load-damping constant & N/A \\
\hline $\mathbf{1 1}$ & $R_{P}$ & Permanent droop & N/A \\
\hline $\mathbf{1 2}$ & $R_{T}$ & Temporary droop & N/A \\
\hline $\mathbf{1 3}$ & $T_{R}$ & Reset time & second \\
\hline
\end{tabular}

\section{B. Simulation parameters}

- Nominal values for the hydroelectric power plant model [1]:

$T_{g}=0.3 ; T_{w}=2 ; \mathrm{M}=10 ; \mathrm{D}=0.8 ; \mathrm{R}_{\mathrm{p}}=5 \%$ $\Delta P_{L}=2 \%$ at $0 \mathrm{~s}$

- $\mathrm{ABC}$ parameters

$>$ Number of variables: $N_{v}=3$

Maximum number of the $\mathrm{ABC}$ iterations: $\mathrm{N}_{\max }=30$

$>$ Population size: $\mathrm{N}_{\mathrm{p}}=8$

$>$ Constraint of the variables: $\left[\begin{array}{ll}x_{\min } & x_{\max }\end{array}\right]=\left[\begin{array}{ll}0 & 20\end{array}\right]$

\section{REFERENCES}

1. P. Kundur. Power system stability and control. McGraw - Hill Education, 1994.

2. Jan Machowski, Z. Lubosny, J. W. Bialek, J. R. Bumby. Power system dynamics: stability and control. Wiley IEEE Press, $3^{\text {rd }}$ edition, 2020. 
3. Vijay Vittal et al. Power system control and stability. Wiley - IEEE Press, $3^{\text {rd }}$ edition, 2019.

4. N.-Khoat Nguyen. A novel improving technology to intelligent controllers - a typical case study for turbine speed regulation of a smart hydropower plant. International Journal of Emerging Trends in Engineering Research, Vol. 8, No. 6, pp. 2388 - 2394, 2020.

https://doi.org/10.30534/ijeter/2020/30862020

5. Thi-Mai-Phuong Dao, Yaonan Wang, Ngoc-Khoat Nguyen. Novel hybrid load-frequency controller applying artificial intelligence techniques integrated with superconducting magnetic energy storage devices for an interconnected electric power grid. Arabian Journal for Science and Engineering, Volume 41, Number 9, pp. 3309-3320, 2016.

6. Peter W. Sauer, M.A. Pai. Power system dynamics and stability. Stipes Publishing Co., $1^{\text {st }}$ edition, 2017.

7. Javad Morsali et al. Appropriate generation rate constraint (GRC) modeling method for reheat thermal units to obtain optimal load frequency controller (LFC). The $5^{\text {th }}$ Conference on Thermal Power Plants (IPGC2014), Iran, pp. 29-34, 2014.

8. Asit Kumar Das et al. Computational intelligence in pattern recognition. Proceedings of CIPR, Springer, 2019.

9. D. Karaboga, B. Basturk. A powerful and efficient algorithm for numerical function optimization: artificial bee colony (ABC) algorithm. Journal of Global Optimization, vol. 39, pp. 459-471, 2007. https://doi.org/10.1007/s10898-007-9149-х

10. Kanendra Naidu, Hazlie Mokhlis, Ab Halim Abu Bakar, Vladimir Terzija. Performance investigation of ABC algorithm in multi-area power system with multiple interconnected generators. Applied Soft Computing, vol. 57, pp. 436-451, 2017.

11. M. Elsisi et al. ABC based design of PID controller for two area load frequency control with nonlinearities. Indonesian Journal of Electrical Engineering and Computer Science, pp. 58-64, 2015.

12. Nour El Yakine Kouba et al. Optimal load frequency control based on artificial bee colony optimization applied to single, two and multi-area interconnected power systems. 3rd International Conference on Control, Engineering \& Information Technology (CEIT), Tlemcen, pp. 1-6, 2015.

13. Zafer Bingul, Oguzhan Karahan. Comparison of PID and FOPID controllers tuned by PSO and ABC algorithms for unstable and integrating systems with time delay. Wiley, 2018.

14. Omdev Dahiya, Kamna Solanki, Sandeep Dalal, Amita Dhankhar. An Exploratory Retrospective Assessment on the Usage of Bio- inspired Computing Algorithms for Optimization. International Journal of Emerging Trends in Engineering Research, Vol. 8, No. 2, pp. 414-434, 2020. https://doi.org/10.30534/ijeter/2020/29822020
15. Ditdit Nugeraha Utama et al. Decision support model for evaluating the level of the implementation effectiveness of 3in1 or ERP systems using fuzzy logic method. International Journal of Emerging Trends in Engineering Research, Vol. 8, No. 7, pp. 3799 - 3803, 2020.

https://doi.org/10.30534/ijeter/2020/144872020 